\title{
FOSTERING EFFICIENT LEARNING IN THE TECHNICAL FIELD OF ROBOTICS BY CHANGING THE AUTONOMOUS DRIVING COMPETITION OF THE PORTUGUESE ROBOTICS OPEN
}

\author{
Valter Costa ${ }^{\mathrm{a}}$, João S. Resende, Patrícia R. Sousa ${ }^{\mathrm{b}}$, Armando Sousa ${ }^{\mathrm{c}}$, Nuno \\ Lau $^{\text {d, Luís Paulo Reis }}{ }^{\mathrm{e}}$ \\ ${ }^{a}$ Faculty of Engineering of the University of Porto (PORTUGAL) \\ ${ }^{b}$ Department of Computer Science Faculty of Science University of Porto (PORTUGAL) \\ ${ }^{c}$ INESC TEC - INESC Technology and Science and FEUP - Faculty of Engineering, \\ University of Porto (PORTUGAL) \\ ${ }^{d}$ Dep. of Electronics, Telecommunications and Informatics, University of Aveiro IEETA \\ - Institute of Electronics and Informatics Engineering of Aveiro (PORTUGAL) \\ ${ }^{e}$ DSI/EEUM - Information Systems Department, School of Engineering, University of \\ Minho LIACC - Artificial Intelligence and Computer Science Laboratory (PORTUGAL)
}

\begin{abstract}
Autonomous Vehicles are a topic of important research. The topic is also visually appealing to public and attractive to educators and researchers. The autonomous driving competition of the Portuguese Robotics Open (PRO) tries to take advantage of this context but concerns arise from lack of participators. In 2017 competition had only 4 teams and this is a worrisome number. Interviews with participants revealed that no participant had a trial track and that the large track size was one of the concerns possibly deterring more teams from participating in this competition. Other worries include the complexity of the minimum setup in this competition and the absence of starting code for beginners. The organizers of the events are also worried about the overall size of the current track. This paper takes advantage of a realistic simulator under Gazebo using Robot Operating System-ROS, studies a new track design, and proposes a track change. The current work proposes a setup with a simulator that may be given by the organization and a new track design to mitigate the presented worries: an open simulator with realistic characteristics under ROS will benefit beginners and will allow entry point
\end{abstract}

DOI: $10.21125 /$ iceri.2017.2049 
code testing, thus reducing entry effort, in turn hopefully appealing to more teams. The simulation world include: a differential steering robot with three cameras as sensors (two pointed down to lane tracking and other pointed up to recognize the signalling panels), a track with two lanes to follow, signalling panels to identify and recognize, and an open space with two circles to perform a 8-shaped circles.In the presented shape, the new track design reduces slightly the occupied space while maintaining the issues the participants are meant to learn: robotics as both a continuous control problem and a discrete event system. Robotics sensory readings from artificial vision system under real time constraints for both $2 \mathrm{D}$ flat image recognition as well as processing of real world imagery seen in 3D perspective. The new track design with small differences to current version is to seam attractive to current participators. The proposed track design ensures that the state machine changing the lane track following mode to the red circle marker following mode is even clearer. That, in turn, clarifies the learning outcomes that participants are intended to learn and who ever learns issues better has an advantage which makes learning attractive to most competitive participants. The simulation and ROS also foster systems thinking, team collaboration and transversal skills such as communication, team cooperation, soft skills, etc.Current challenges already include obstacle avoidance and lane changing and such issues are not approached here. The suggestion is that these challenges are not to be changed, thus keeping this part of the challenges not altered and issues related unaltered: these challenges make sure that all cameras are needed and increase complexity of the high level state machine but don't introduce as much novelty as the rest of the competition.It is hopped that the new track design is visually appealing to educators, learners/participants and to general public but rule changes are always a delicate issue as attracting new teams may eventually deter previous participators.

Keywords: Autonomous Driving, State Machines, Gazebo, ROS, Mobile Robotics, Educational Robotics 